that this is so. For example, their $x, y$ distribution in the field follows the same pattern as that shown by the very blue stars, whereas the field stars $(0 \cdot 5<B-V$ $<1 \cdot 5)$ are uniformly distributed over the plate.

Many persons on the staff of the Royal Greenwich Observatory took part in the work. I am particularly indebted to H. M. Astronomer at the Cape, Dr. R. H. Stoy, for the magnificent cooperation which he has set up between his Observatory and Greenwich, and to Dr. A. D. Thackeray, the Radcliffe Observer, for help in many directions.

\title{
Discussion
}

Tifft: How large an area in the Clouds can be eventually studied in this manner?

Woolley : Long exposure plates were taken with the Cape astrograph which cover all of the LMC; some of these are not of such good quality as the one we have used. We certainly plan to continue the work but we feel it necessary to determine $B$ and $V$ for all the stars whose proper motion we measure, in order to be able to interpret the results, and we have now asked Dr. Stoy to organize the setting up of suitable photoelectric sequences.

Arp : Are there any suitable first epoch plates available for the SMC?

Woolley: Unfortunately, no.

Bok: Would you please consider using old plates now at Harvard for measurement of proper motions? The old Bruce plates and the Metcalf plates should yield annual proper motions to within \pm 0 ". 010 - possibly considerably better. These old plates together with new ones can be used effectively now for the elimination of foreground stars.

Woolley: Dr. Menzel has agreed in principle to lend us these plates and I hope to arrange for one of the senior members of the Greenwich staff to go and inspect them.

Arp: Dr. Woolley reports that in the $V=13$ to 14 mag range in the LMC there is an absence of members with $B-V$ from 0.6 to $1 \cdot 5$. This agrees exactly with the colour-magnitude diagram of NGC 330 in the SMC. I would expect, however, that as one penetrates to the $V=16$ mag range the majority of evolved giants would be precisely in this colour range.

Woolley: I hope to present evidence on Thursday which shows that the stars down to $V=16^{\mathrm{m}}$. 5 in the colour range $0.5<(B-V)<1.5$ are still not members of the LMC.

Gascoigne: Both Melbourne and Yale-Columbia should have suitable first epoch plates of the Clouds. Melbourne in particular have some astrograph plates of the Large Cloud taken last century. Perhaps Mr. Harley Wood knows something about these.

Wood: The Melbourne chart plates have been at Greenwich. They do not reach magnitudes as faint as those discussed by the Astronomer Royal.

\section{THE SURFACE DISTRIBUTION`OF CLUSTERS, PLANETARY NEBULAE, SUPERGIANT M STARS, AND CARBON STARS IN THE LARGE MAGELLANIC CLOUD}

\author{
B. E. WesterLund \\ Mount Stromlo Observatory
}

\section{The Clusters}

Some years ago the plates used for the extended Uppsala-Mount Stromlo Atlas of the Magellanic Clouds were searched for clusters in order to compare the optical extension of the Large Cloud with the one defined by the radio-astronomical 
observations of neutral hydrogen. Slightly over 150 clusters were identified and good agreement between the radio and optical boundaries was noted (Westerlund, unpublished data).

The limiting magnitude of the visual Atlas plates is about $V=18 \cdot 2$ and it was found likely that a large number of clusters may have escaped detection.

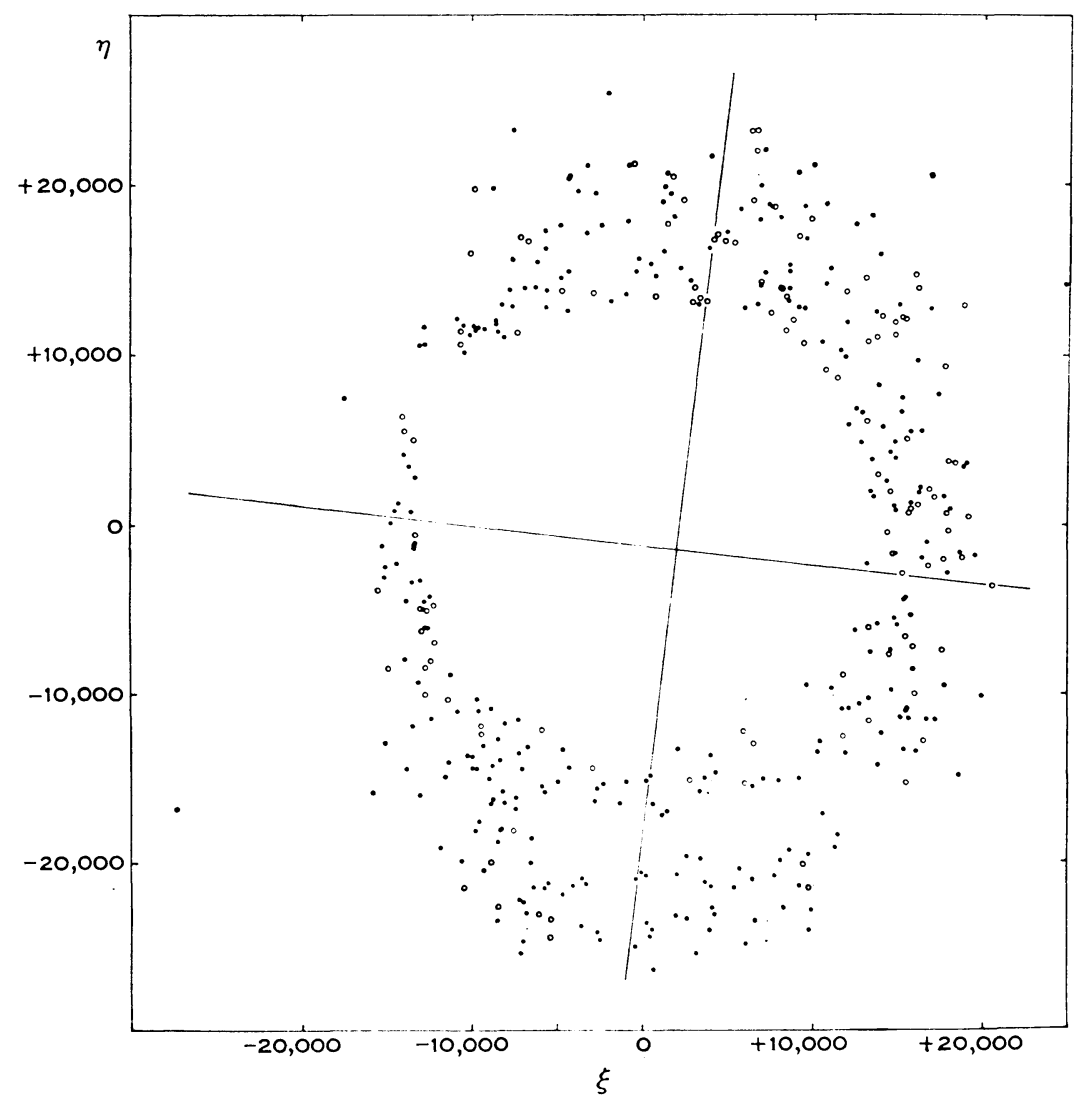

Fig. 1.-The distribution of the clusters in the outer parts of the LMC. Dots represent definite clusters, open circles possible clusters. Wesselink's coordinate system is used.

The survey has now been repeated by Lynga and Westerlund (1963) using a more extensive plate material gathered by Lynga with the Schmidt telescope of Uppsala Southern Station. The new material consists of blue-sensitive plates (Eastman Kodak 103aO+Schott GG13 filter) reaching a limiting magnitude of $B=20$ mag and of red-sensitive plates (Eastman Kodak 103aF+Schott GG14) reaching a limiting magnitude of $V=19 \mathrm{mag}$.

We have avoided the inner crowded regions of the Cloud (inside $r=3^{\circ}$ ) and have identified 480 clusters in the outer regions. The distribution of about 420 of these objects is shown in Figure 1, where all the clusters outside $r=13,000$ in the 
Wesselink coordinate system (Wesselink 1959) are plotted. In equatorial coordinates, most of the clusters are found between R.A. $=4^{\mathrm{h}} 25^{\mathrm{m}}$ and $6^{\mathrm{h}} 35^{\mathrm{m}}$ and dec. $-62 \cdot 5$ and $-76^{\circ}$.

The observed cluster system is obviously elliptical and has a well-defined centroid at $\xi=2000, \eta=-1500\left(5^{\mathrm{h}} 32^{\mathrm{m}},-69^{\circ} .3\right)$. The position angle of the major axis is $7 \pm 10^{\circ}$ (estimated error). The axial ratio is close to $1 / \sqrt{ } 2$, indicating a tilt of $45^{\circ}$ of the Cloud, if the real system is circular.

Most of the clusters appear to be fairly old open (or galactic) clusters and may be expected to form a disk system. One outstanding feature is the complete lack of objects in an extended area around $\eta=-18,000$ (declination $-74^{\circ}$ ). This break in the cluster system agrees well in position with a deficiency in stars found by de Vaucouleurs (1955) from long-exposed photographs. The cluster system south of this gap agrees well in extension with an arm drawn by de Vaucouleurs. It appears likely that a detailed study of the cluster system will give important information about the distribution of the older Population I in the Cloud.

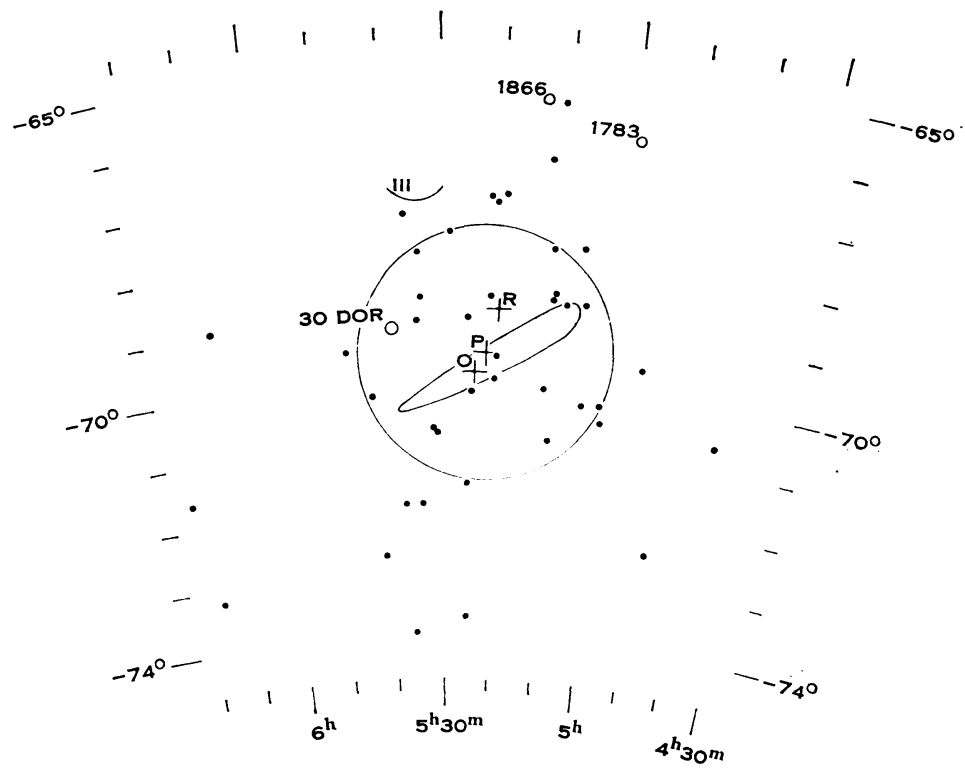

Fig. 2. - The distribution of planetary nebulae in the LMC. Coordinates are for the epoch $1975 \cdot 0$. The radio centre $(R)$, the optical centre $(\mathrm{O})$ of the core, and the centre of the planetary nebulae (P) are marked. The circle has a radius of $2^{\circ}$ and is centred on $P$.

\section{The Planetary Nebulae}

The planetary nebulae in our Galaxy are generally assumed to form a disk system with a high concentration towards the galactic centre (Minkowski 1951; see, however, Perek, this volume, paper 57). The distribution of the identified 42 planetary nebulae in the Large Cloud may, therefore, be expected to give some information about the position of the true centre of the Cloud. Admittedly, the identified objects are the most luminous ones and constitute probably only a tenth of the total number 
of planetaries in the Cloud (see Westerlund, this volume, paper 65). The situation in our Galaxy is, however, similar as far as the number of identified planetaries is concerned.

Figure 2 shows the distribution of the planetary nebulae in the Large Cloud. They define a fairly circular system with the centroid at $5^{\mathrm{h}} 22^{\mathrm{m}} \pm 3^{\mathrm{m}},-69^{\circ} 5 \pm 0 \% 4$; 21 of the planetaries fall inside a circle with a radius of $2^{\circ}$ (approx. $2 \mathrm{kpc}$ ). For comparison the optical geometrical centre of the core $(0)$ at $5^{\mathrm{h}} 24^{\mathrm{m}},-69^{\circ} .8$ and the centre of symmetry of the HI rotation curve $(\mathrm{R})$ at $5^{\mathrm{h}} 20^{\mathrm{m}},-68^{\circ} 8$ are marked (Buscombe, Gascoigne, and de Vaucouleurs 1955) in the figure. It may be recalled, in this connection, that the centroid of the outer cluster system is at $5^{\mathrm{h}} 32^{\mathrm{m}},-69^{\circ} 3$ (see above) and that the centroid of the HI radiation is at $5^{\mathrm{h}} 34^{\mathrm{m}},-68^{\circ} .5$ (Buscombe, Gascoigne, and de Vaucouleurs 1955). It appears impossible to define a unique centre for all the objects in the Cloud. However, it is unlikely that the true centre can be situated outside the bar with its enormous population of stars. Although there is no reason for a visible centre in an irregular galaxy, I find it tempting to suggest that the object NGC 1916 at $5^{\mathrm{h}} 19^{\mathrm{m}},-69^{\circ} 4$ in the middle of the bar be considered as the possible centre. This object has been classified as a globular cluster, but it is not listed among Hodge's red clusters (Hodge 1960). Large-scale photographs of the object in infrared, red, yellow, and blue light show all a very bright nucleus. So far, it has not been possible to resolve its central parts into stars. The nucleus has a diameter of about $7^{\prime \prime}$ on the infrared plates and remains approximately the same in other colours.

\section{The $M$ Supergiants and the Carbon Stars}

The major part of the Large Cloud has now been observed for the classification of the red stars in the Case infrared system. The results for four regions in the central parts of the Cloud have been published (Westerlund 1960). At present over 600 stars have been identified as possible M0-M4 supergiants and over 400 objects have been classified as carbon stars. The number of late $M$ stars appears very large. The detailed study of this group over the whole Cloud remains to be done.

Figure 3 shows the distribution of the early M stars (dots) and the carbon stars (open circles) in the Large Cloud. The full-drawn line marks the outer boundary of the region where the identification of the carbon stars is assumed to be complete - to about $I=14 \mathrm{mag}$. Most of the early $M$ supergiants appear to have infrared apparent magnitudes brighter than $I=12 \mathrm{mag}$. The survey for these objects is therefore complete over a larger area. From the comparison field in the lower right corner of Figure 1 and from the general distribution of $\mathbf{M}$ stars in the south-east part of the surveyed region we conclude that between one and two $M$ stars per square degree may be galactic objects. All $M$ stars brighter than $I=9$ mag are most likely foreground stars. Only one apparently bright carbon star has been found, at about $7^{\mathrm{h}},-\mathbf{7 3}^{\circ}$ (dot in circle). It is assumed to be a galactic object.

The early $M$ supergiants show a strong concentration to regions rich in blue stars but appear to avoid the regions rich in nebulosity. Constellation III has about $25 \mathrm{M}$ stars per square degree; the regions around 30 Doradus have a surface density of near $50 \mathrm{M}$ stars per square degree and two other associations, at $4^{\mathrm{h}} 55^{\mathrm{m}},-69 \% 5$ 

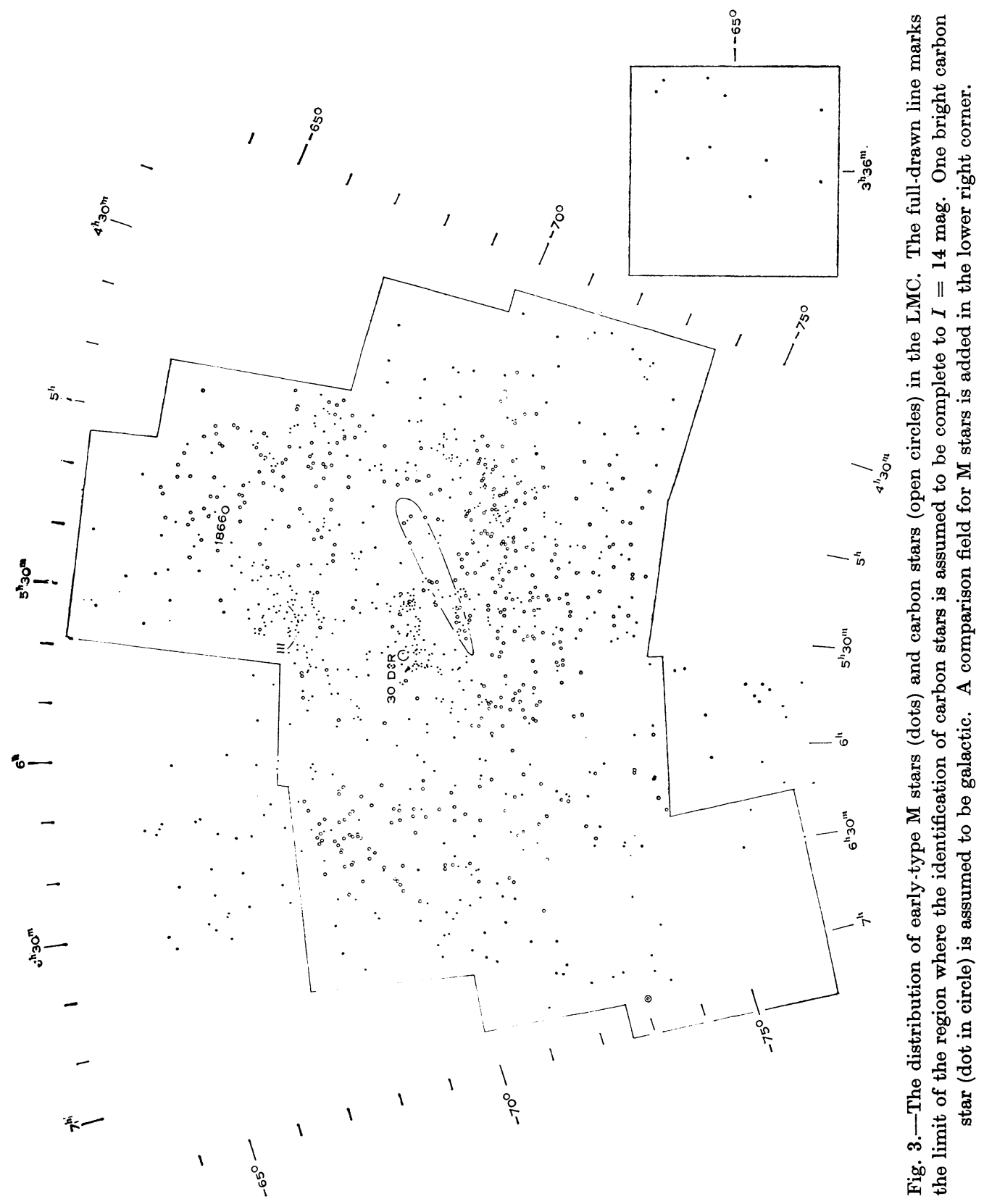
and at $5^{\mathrm{h}} 07^{\mathrm{m}},-70^{\circ} 7$, have a similar density. Outside these associations the density is about $4 \mathrm{M}$ stars per square degree over the whole field $\left(4^{\mathrm{h}} 30^{\mathrm{m}}\right.$ to $6^{\mathrm{h}} 30^{\mathrm{m}}$ in R.A. and $-64^{\circ}$ to $-74^{\circ}$ in dec.). If the early $M$ supergiants are assumed to be stars recently evolved away from the main sequence their associations must be among the youngest in the Cloud. If the stellar evolution always follows the same pattern we may expect to find still younger associations among those without $M$ supergiants. Their main sequences must then end within 1.5 magnitudes (in $V$ ) from the turn-off point of the associations containing $M$ stars.

In a previous paper (Westerlund 1960) it was suggested that the carbon stars in the Large Cloud may be typical for the bar. It may be recalled that only nine objects were identified at that time. Improved plate material and the extension of the survey to outer regions of the Cloud have disproved this conclusion. It may now be concluded that the carbon stars avoid the central regions as well as the regions rich in nebulosity and in blue stars. As in our Galaxy they appear to form clusterings, possibly in arms as indicated by the sizes of the clusterings. It is of interest to note the good agreement between the clusterings of the carbon stars and the structural features noted by de Vaucouleurs (1955) on long-exposed photographs of the Large Cloud. It appears likely that the carbon stars belong to the old Population I, although they appear to define galactic spiral arms better than the old open clusters, and to form more well-defined groupings in the Cloud. Definite conclusions will have to await (1) the extension of the survey for carbon stars to the region rich in clusters south of the gap at $-74^{\circ}$ (see above), and (2) the determination of magnitudes and colours for some clusterings of carbon stars in the Magellanic Clouds and in the Galaxy.

\section{References}

Buscombe, W., Gascoigne, S. C. B., and de Vaucouleurs, G. (1955).-Aust. J. Sci. 17 : No. 3, Suppl.

HodGe, P. W. (1960).-Ap. J. 131 : 351-7.

LyNGA, G., and WesterLund, B. E. (1963).-M.N. 126: (in press).

Mrnkowski, R. (1951).- “The Structure of the Galaxy: a Symposium." [Ann Arbor, Mich. 1950.] Publ. Obs. Univ. Mich. 10: 25-32.

DE VAUCOULEURS, G. (1955).-A.J. 60: 126-40.

Wesselink, A. J. (1959).-M.N. 119: 576-9.

Westerlund, B. (1960).-Uppsala Obs. Ann. 4 : No. 7.

\section{Discussion}

Ambartsumian: What is the limit of apparent magnitude of early M-type stars you have shown in your distribution diagram?

Westerlund: About 12 or 12.5 in the infrared magnitude system.

de Vaucouleurs: The carbon stars clearly follow the path of the faint outer loop seen on blue-light photographs, especially near $6^{\mathrm{h}} 15^{\mathrm{m}}$ between -68 and $-73^{\circ}$. It is the first time that individual objects have been traced in this outer arm. The brighter inner arms are also clearly marked by the M- and especially C-star distribution.

Arp : In the cluster distribution there is an absence of clusters in a lane at negative $\xi$ which agrees with a paucity of star counts. Is this caused by obscuration or positive increase in star density?

Westerlund: It appears to me to be due to a real increase in stellar and cluster density.

Thackeray: The OB stars in the HDE Catalogue already define a rather elliptical distribution somewhat similar to those shown by the clusters. 
With regard to the planetaries I was very interested to see that Dr. Westerlund finds a centroid within the bar, while the centroid of $\mathrm{HI}$ radiation (which agrees with the centre of both radio and optical rotation) lies well outside the bar.

Westerlund: The older population may define a different centroid from that of the gas and the young stars.

Perek: The planetaries do not seem to be very strongly concentrated. Of course, the giant planetaries in the LMC may have a different distribution in space from galactic planetaries.

Tifft: The bar population is probably rather old compared with most of the other types of objects we have been considering. I would not be surprised to see significant shifts in system centroids as a function of age of the systems in view of the rather irregular dynamics of the system.

de Vaucouleurs: The cluster distribution on the south side shows also clearly the part of the outer loop near $-75^{\circ}$ between $4^{\mathrm{h}} 50^{\mathrm{m}}$ and $6^{\mathrm{h}} 10^{\mathrm{m}}$ which is not covered by the $\mathrm{C}$ stars search.

Ambartsumian: I have noted a very strong concentration of M-type stars on your diagram, just within 1 degree from 30 Doradus. Does it coincide with any concentrations of objects of some other type?

Westerlund: It coincides well with groupings of blue stars, generally where there is no or little HII emission visible.

Tifft: Could you say roughly what the apparent visual luminosity of the carbon star group is, and how much spread is seen?

Westerlund: Approximately $15 \cdot 7$ with a spread of about $\pm 0 \cdot 5$ mag.

\title{
54. CONTINUUM RADIO EMISSION FROM THE MAGELLANIC CLOUDS
}

\author{
D. S. Mathewson and J. R. Healey \\ CSIRO Radiophysics Laboratory, Sydney
}

\section{Radio Emission from "Normal" Galaxies}

Before discussing the results of the radio observations of the Magellanic Clouds in detail, it is worth while to briefly review some of our current ideas about radio emission from "normal" galaxies which would be the classification given to the Clouds by radio astronomers. A "normal" galaxy has no striking visual peculiarity and has a much lower ratio of radio to light flux than the "radio" galaxy.

Hanbury Brown and Hazard (1961b) found that in general the ratio of radio to light flux is less for irregular galaxies than for spiral galaxies. This was supported by the surveys of Mills (1959) and Heeschen (1961). A recent survey of 37 "normal" galaxies by Mathewson and Rome $(1963 b)$ using the 210-foot reflector of the ANRAO at $1410 \mathrm{Mc} / \mathrm{s}$ has also confirmed this result. The magellanic-type galaxies NGC 55 and 6822 were amongst the irregular galaxies included in this survey. Table 1 gives radio indices at $1410 \mathrm{Mc} / \mathrm{s}$ for these two galaxies together with those for the Large and Small Clouds obtained by integration of the $1410 \mathrm{Mc} / \mathrm{s}$ isophotes in Figures 2 and 3. $R$, the radio index, is defined as $m_{r}-m_{p g}$, where $m_{r}$ is the radio magnitude at $1410 \mathrm{Mc} / \mathrm{s}$. The $16 \mathrm{Sc}$ galaxies detected in this survey had a mean radio index at $1410 \mathrm{Mc} / \mathrm{s}$ of $+3 \cdot 3$ with an r.m.s. deviation of $0 \cdot 6$.

Until now our ideas about the broad-scale structure of radio emission from "normal" galaxies were formed from observations of the Galaxy and M31. Analysis 